\title{
Hardware Implementation of OFDM system to reduce PAPR using Selective Level Mapping on FPGA
}

\author{
Rajesh S. Bansode ${ }^{1}$, Dr. B. K. Mishra ${ }^{2}$ and Aqsa M. Temrikar ${ }^{3}$ \\ ${ }^{I}$ (I.T Department, Thakur College of Engineering and Technology, India) \\ ${ }^{2}$ (Principal, Thakur College of Engineering and Technology, India) \\ ${ }^{3}$ (M.E student, Thakur College of Engineering and Technology, India)
}

\begin{abstract}
OFDM is a modulation as well as multiplexing technique which is widely used in various high speed mobile and wireless communication systems because of its capacity of ensuring high level robustness against interference. In this paper the design and implementation of OFDM system along with SLM implementation to reduce PAPR[6]is illustrated and a detailed simulation of the OFDM system with 16-QAM. OFDM transceiver is implemented using FPGA Spartan6 kit. The hardware results show a detailed study of RTL schematics and Test Bench. In this paper, the software simulation results show $2 d B$ reduction in the peaks. Keywords - Field Programmable Gate Array, Matlab Simulink, Orthogonal Frequency Division Multiplexing, Peak-to-Average Power Ratio, Selective level Mapping and Xilinx
\end{abstract}

\section{INTRODUCTION}

Orthogonal Frequency Division Multiplexing (OFDM) is an attractive multicarrier technique for mitigating the effects of multipath delay spread of radio channel, and hence accepted for several wireless standards as well as number of mobile multimedia applications. Alongside its advantages such as robustness against multipath fading, spectral efficiency and simple receiver design, OFDM has two major limitations.

One of these is its sensitivity to carrier frequency offsets (CFO) caused by frequency differences between the local oscillators in the transmitter and the receiver, phase noise and the other is high peak to average power ratio (PAPR). This high PAPR is due to the summation of sinc-pulses and non-constant envelope. Therefore, RF power amplifiers (PA) have to be operated in a very large linear region. Otherwise, the signal peaks get distorted, leading to intermodulation distortion (IMD) among the subcarriers and out-of-band radiation. A simple way to avoid is to use PA of large dynamic range but this makes the transmitter.

The paper aims at successful implementation of the transceiver on a FPGA which would pave a way towards developing an OFDM system which resolves the issue of high PAPR. Simulation results using System Generator and Matlab/Simulink and XILINX tools have been given.Finally it aims at development of a complete system which then results in robust, maximum throughput, highly scalable wireless LAN network.

\subsection{General OFDM Block Diagram Descriptions}

\section{OFDM, PAPR AND SLM}

\subsubsection{OFDM Transmitter}

The model considered for the implementation of the OFDM transmitter is the shown below and basically consist of [13]

Serial to parallel converter, Constellation modulator, IFFT block, Parallel to serial converter, Digital to Analog converter, Selective Level Mapping

\subsubsection{OFDM Receiver}

OFDM receiver is the shown below and basically consist of [13]

Analog to digital converter, Serial to parallel converter, Cyclic prefix removal, FFT block, M-QAM decoder, Parallel to serial converter. 


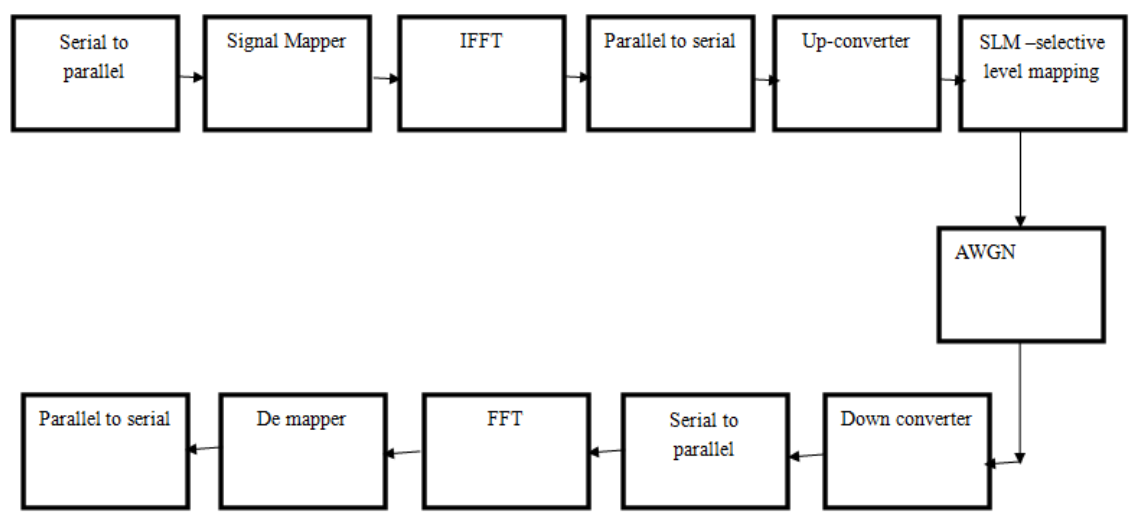

Fig 2.1. Block diagram of an OFDM system

\subsection{Peak- to- average Power ratio-PAPR}

The major disadvantage of using several subcarriers in parallel using IFFT is the highly non-constant envelope of the transmit signal, making OFDM very sensitive to nonlinear components in the transmission path. A key component is the high power amplifier (HPA). Due to cost, design and most importantly power efficiency considerations, the HPA cannot resolve the dynamics of the transmit signal and inevitably cuts off the signal at some point causing additional in-band distortions and adjacent channel interference. The power efficiency penalty is certainly the major obstacle to implement OFDM in low-cost applications. Moreover, in powerlimited regimes determined by regulatory bodies, the average power is reduced compared to single-carrier systems reducing in turn the range of transmission. The power control problem motivates further research since it touches on many of the advantages that originally made OFDM transmission popular, i.e. spectral efficiency and implementation issues.

In OFDM systems, a fixed number of successive input data samples are modulated and then jointly correlated together by use of IFFT at the transmitter side. IFFT processes signals to produce orthogonal data sub-carriers . Mathematically, IFFT combines all the input signals to produce each one of the output symbols.

. The signal processing by IFFT in the OFDM transmitter changes the statistical distribution of signals from uniform-to-Gaussian. Therefore, the dynamic range of the OFDM output envelope is most often higher than that of the single-carrier systems. However, PAPR is widely used to evaluate the dynamic range of the output envelope. The PAPR (in $\mathrm{dB}$ ) is defined by the following equation [14]

$$
\text { PAPR }=10 \log _{10}\left\{\frac{P_{\text {peak }}}{P_{\text {avg }}}\right\}=10 \log _{10} \frac{\max \left[|x(n)|^{2}\right]}{E\left[\left|x_{n}\right|^{2}\right]}
$$

where $P_{\text {peak }}$ represents peak output power, $P_{\text {average }}$ is average output power. $E[\cdot]$ denote the expected value, $x_{n}$ represents the transmitted OFDM signals which are obtained by taking IFFT operation on modulated input symbols $\mathrm{X}_{\mathrm{k}} \cdot \mathrm{X}_{\mathrm{n}}$ is also expressed as[12]

$$
x_{n}=\frac{1}{\sqrt{N}} \sum_{K=0}^{N-1} X_{k} W_{N}^{n k}
$$

\subsection{Selective Level Mapping - SLM}

Selective level Mapping (SLM)[6] was first proposed in 1996 to reduce PAPR in OFDM systems. The system block diagram of SLM is shown below. At first, the input information is divided into OFDM data block $\mathrm{X}$, which consists of $\mathrm{N}$ symbols, by the serial-to parallel (S/P) conversion and then data block $\mathrm{X}$ is multiplied carrier wise with each one of the $\mathrm{U}$ different phase sequences $\mathrm{B}(\mathrm{u})$, resulting in a set of $\mathrm{U}$ different OFDM data. In the SLM[8] algorithm the data source denoted as $\mathrm{X}$, is multiplied by the $\mathrm{U}$ different sets of phase factors/masks, element-wise to produce U different copies of $\mathrm{X}$,

$$
\mathrm{Xu}=\mathrm{BuX} \quad \mathrm{u}=1,2, \ldots, \mathrm{U}
$$

where $\mathrm{U}$ is the design parameter in SLM. In general, more reduction in PAPR is likely to achieved when $\mathrm{U}$ in-creases.[12]. In addition, $\mathrm{Bu}$ is defined as:

$$
\mathrm{Bu}=[\mathrm{Bu}, 1 \mathrm{Bu}, 2 \mathrm{Bu}, 3 \ldots \mathrm{Bu},(\mathrm{N}-1)]
$$


Then all $\mathrm{U}$ alternative data blocks (one of the alternative sub-carrier sequences can be the unchanged original one) are transformed into time domain to get transmit OFDM symbol where $\mathrm{N}$ represents the number of subcarriers in IFFT and $\mathrm{Bu}, \mathrm{k}$ is given by:

$$
\mathrm{Bu}, \mathrm{k}=\mathrm{e}^{\mathrm{j} \phi} \mathrm{u}, \mathrm{k} \quad \mathrm{k}=0,1,2, \ldots, \mathrm{N}-1
$$

After multiplying $\mathrm{X}$ with the phase factors, each $\mathrm{Xu}$ is processed by IFFTs and its PAPR is then computed and compared with the others. The resulting signal that yields the lowest PAPR is subsequently chosen for transmission. In addition, the Bopt which implies the optimal Bu that produces the lowest PAPR has to be transmitted to receiver as a side information[14]. The receiver will then use Bopt to recover the data source, $\mathrm{X}[6]$.

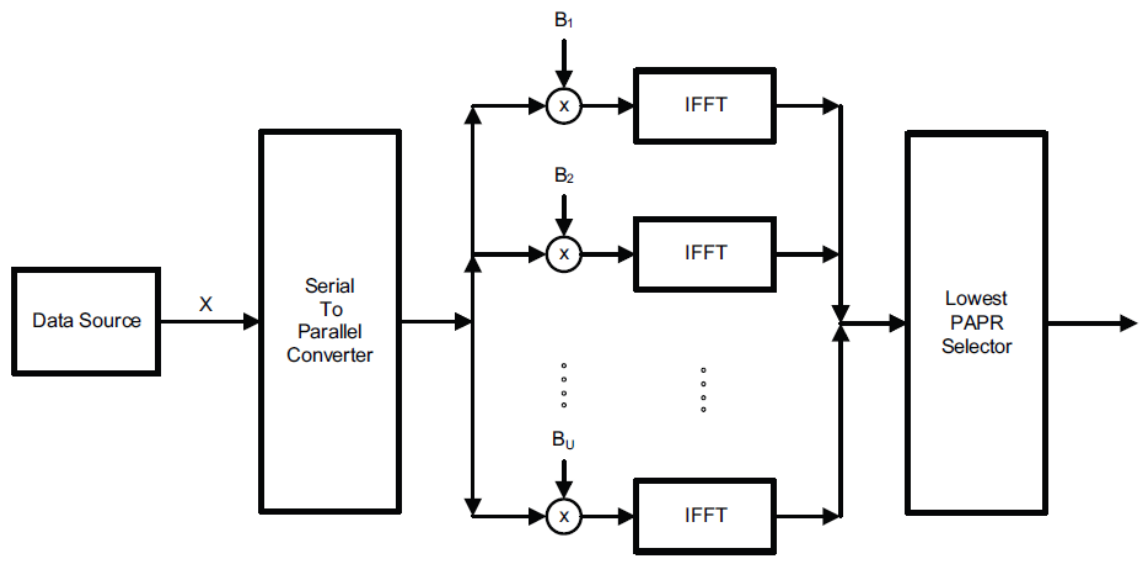

Figure 2.2 Block diagram of SLM[6]

\section{Transmitter Design}

\section{IMPLEMENTED OFDM MODEL}

In the design presented, block length of 8 points and 4 different phase sequences are used. Transmitter subsystem design contains 4 independent OFDM modulators. In the modulator, the input data is multiplied by a look-up table from ROM before giving it to actual OFDM modulation process. This lookup table holds the phase sequences for each block. The output of each block is given to a PAPR calculator and selector. It transmits the data block with lowest PAPR and also transmits the selected block number.

\section{Receiver Design}

The receiver is a normal OFDM receiver with a phase sequence selector block. The data stream is first given to FFT block for decoding. Then the data stream is passed to a lookup table multiplier which multiplies data with a phase sequence as indicated by the selected block number from transmitter.

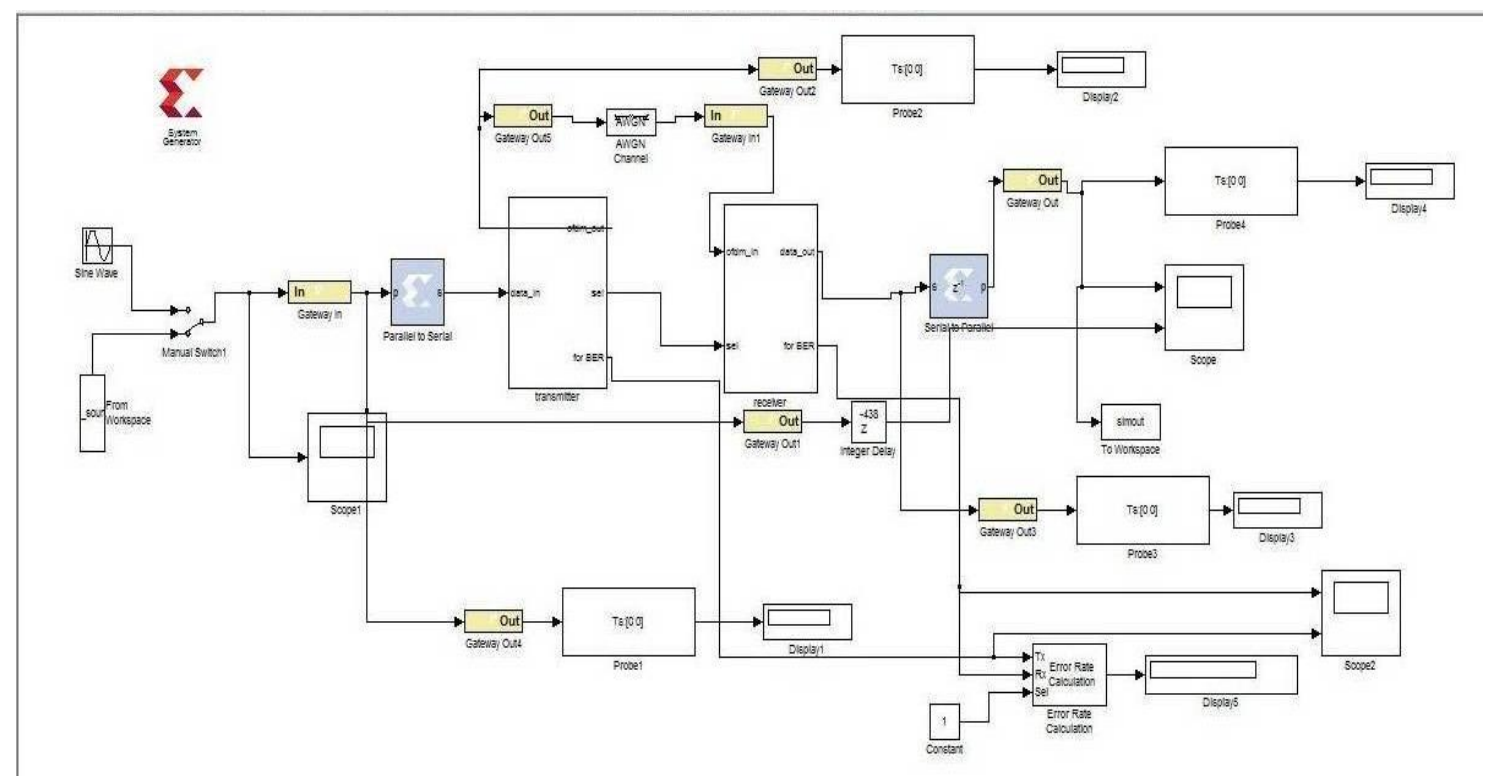

Figure 3.1 Block diagram of the designed system 


\section{Simulation Results}

Table 4.1 indicates PAPR values with SLM and without SLM. Last column shows Crest Factor $(\sqrt{ } \mathrm{K})$. It is evident from the table that PAPR reduction of about $2 \mathrm{~dB}$ is obtained using 64 carriers. Varying difference in PAPR is obtained using 2,4,8 and 64 number of carrier.

PAPR reduction of nearly $1.6 \mathrm{~dB}$ is obtained using 2 carriers. Higher reduction of PAPR is obtained using 8 carriers

\section{TABLE 4.1 PAPR obtained with and without}

SLM, CRESTFACTOR

\begin{tabular}{|c|c|l|c|c|}
\hline $\begin{array}{c}\text { Number } \\
\text { of Sub- } \\
\text { carriers }\end{array}$ & $\begin{array}{c}\text { PAPR } \\
(\mathrm{K}) \\
\text { without } \\
\text { SLM } \\
\text { in dB }\end{array}$ & $\begin{array}{l}\text { PAPR(K) } \\
\text { with } \\
\text { SLM in } \\
\mathrm{dB}\end{array}$ & $\begin{array}{c}\text { Difference } \\
\text { in PAPR }\end{array}$ & $\begin{array}{c}\text { Crest } \\
\text { Factor } \\
=\sqrt{ } \text { PAPR } \\
=\sqrt{\mathrm{K}}\end{array}$ \\
\hline 2 & 12.61 & 11.01 & 1.6 & 1.264 \\
\hline 4 & 25.22 & 24.01 & 1.21 & 1.1 \\
\hline 8 & 37.83 & 35.03 & 2.77 & 1.67 \\
\hline 16 & 50.45 & 48.89 & 1.56 & 1.24 \\
\hline 32 & 63.06 & 62.01 & 1.59 & 1.024 \\
\hline 64 & 75.76 & 73.76 & 2 & 1.414 \\
\hline
\end{tabular}

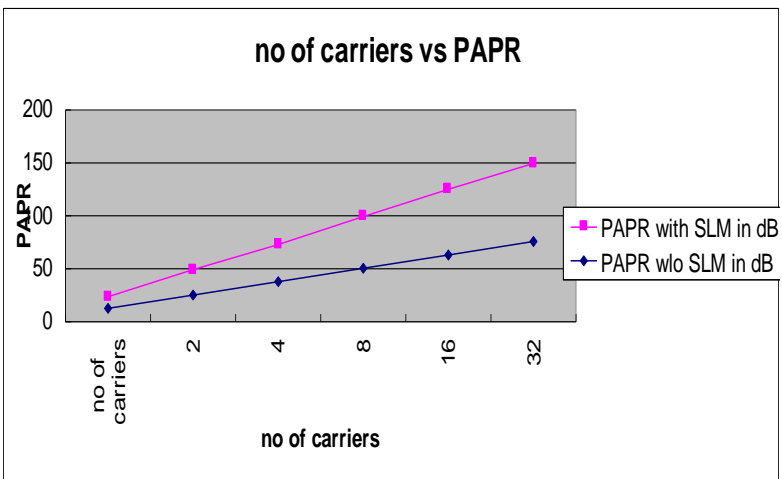

Fig 4.1 Number of carriers v/s PAPR

As number of subcarriers increase the PAPR increases. Implementing the proposed technique PAPR can obtain reduction in PAPR.

\section{Hardware Implementation}

The hardware simulation[24] of the model was carried out in step by step manner. Small models of the components of the model were made and their outputs on the wavescope were observed. RTL logic and test benches of the intermediate models were obtained.

The detailed description of the intermediate blocks is shown below:

\subsection{Experiment No.1 - Serial to Parallel}
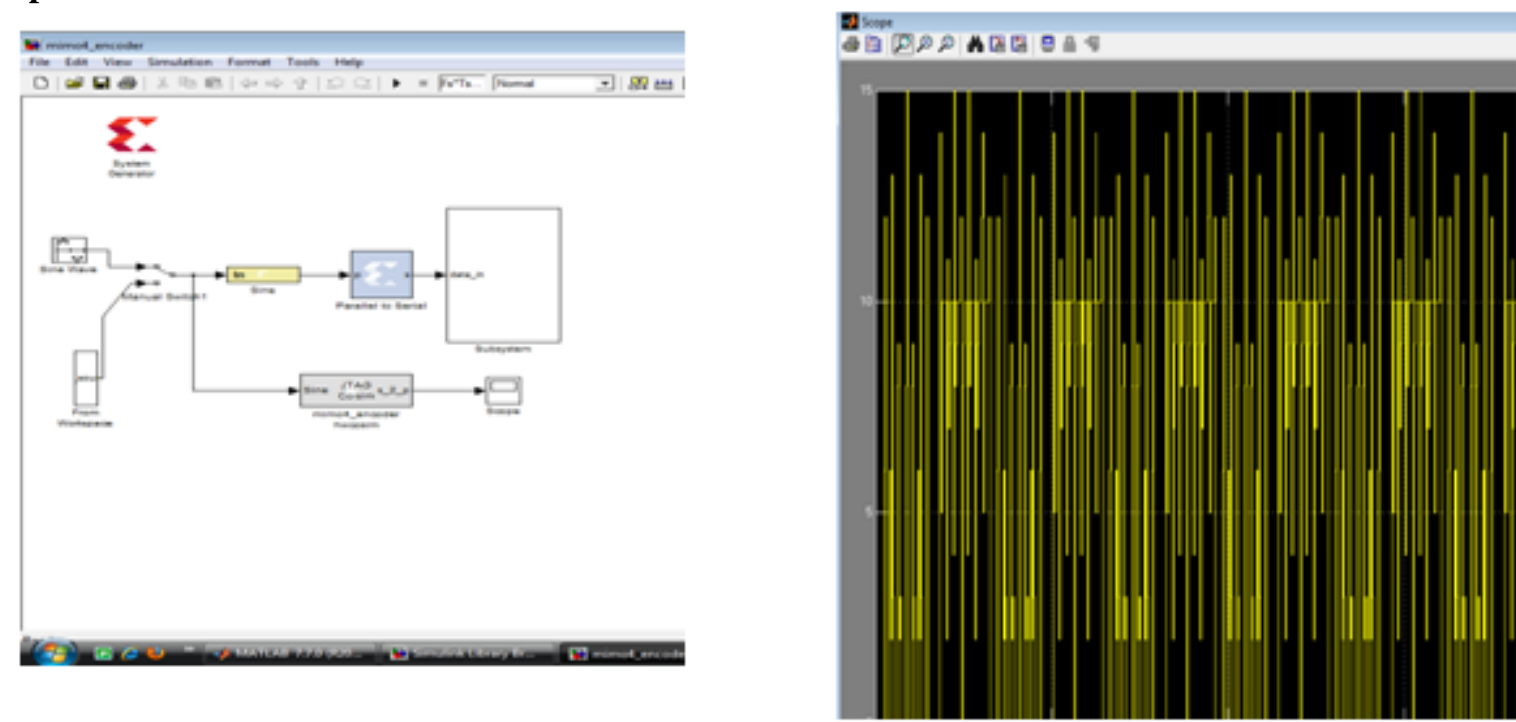

Fig 5.1 (a) hardware model of serial to parallel (b) wavescope results of FPGA simulations

Fig 5.1 (a) hardware model of serial to parallel (b) wavescope results of FPGA simulations The hardware model using JTAG is shown in fig 5.1(a) and the wavescope results of the same is shown in fig 5.1(b) . The RTL schematic for the above model is as shown below. 

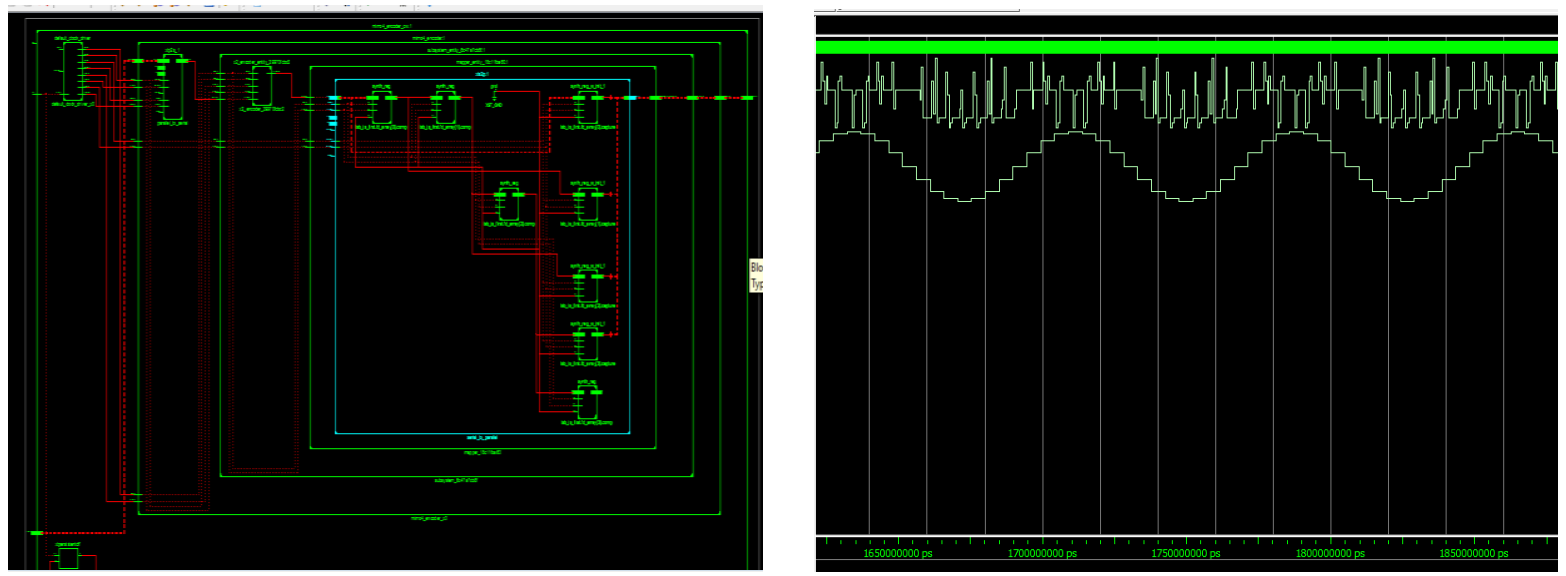

Fig 5.2 (a) RTL Schematic (b) Test Bench

This completes the hardware simulation of serial to parallel

5.2 Experiment no. 2 - FFT block

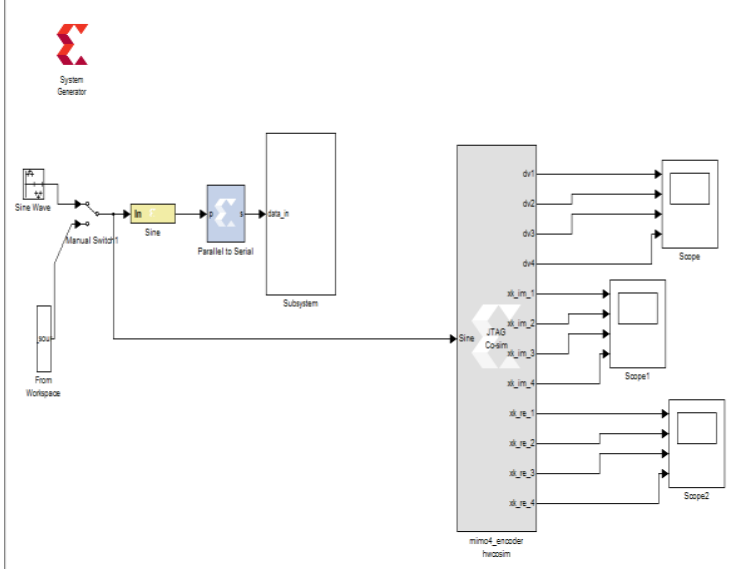

Fig 5.3 (a) hardware model of FFT
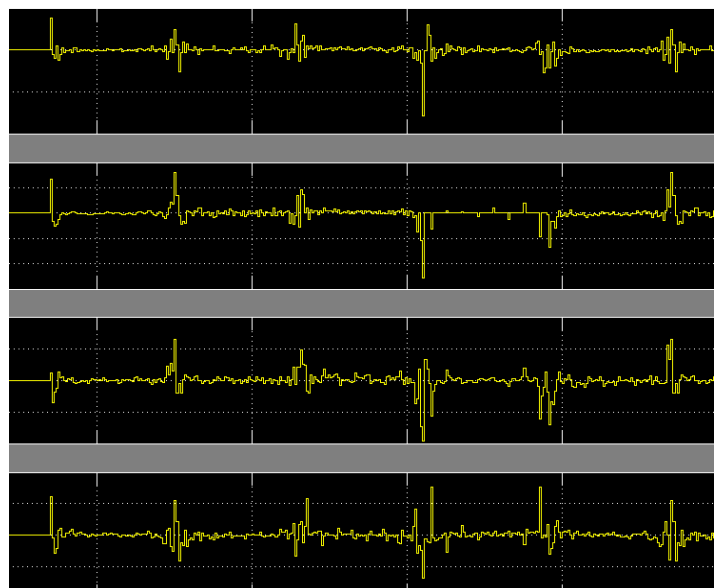

(b) wavescope results of FPGA simulations

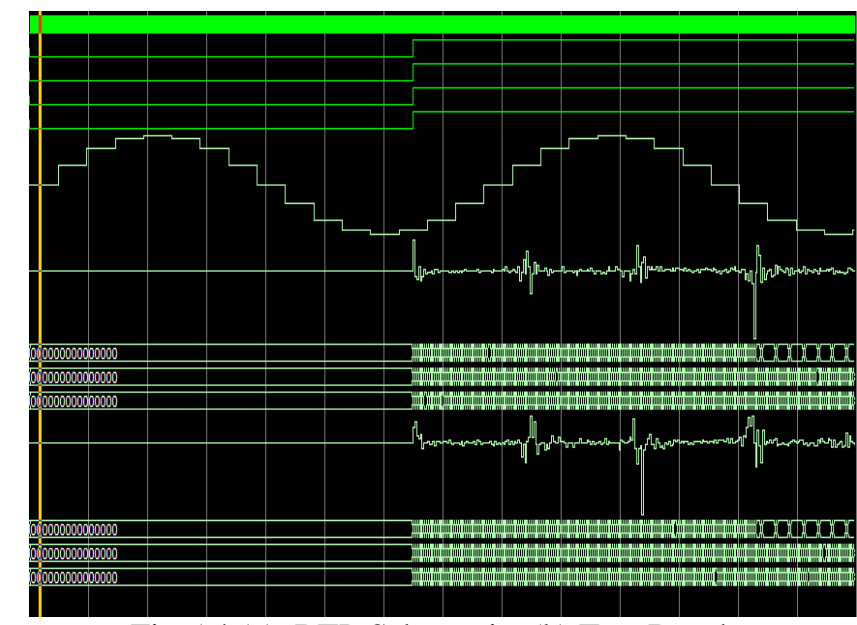

Fig 5.4 (a) RTL Schematic (b) Test Bench

1.3 Experiment No. 3 - Hardware Simulation of Entire Model

Using XILINX and modelsim the developed simulink model is converted into its JTAG equivalent.[2]

Internal simulator of XILINX, i-sim can also be used in place of modelsim.[20]

The hardware implementation of the simulink model is done using JTAG. The process of developing such a hardware is called Hardware-In-Loop. 


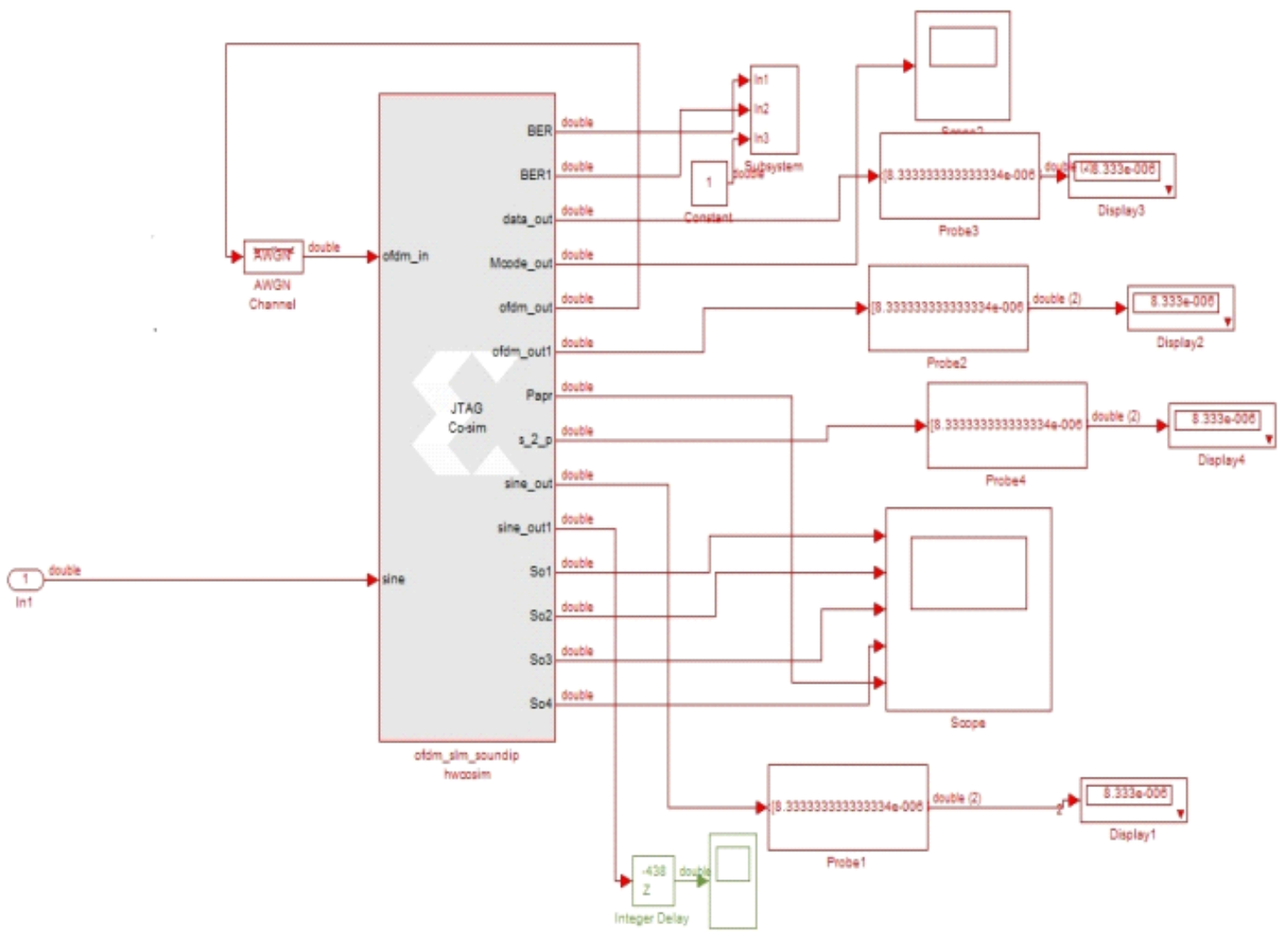

Fig 5.5 JTAG-Hardware Co-sim block
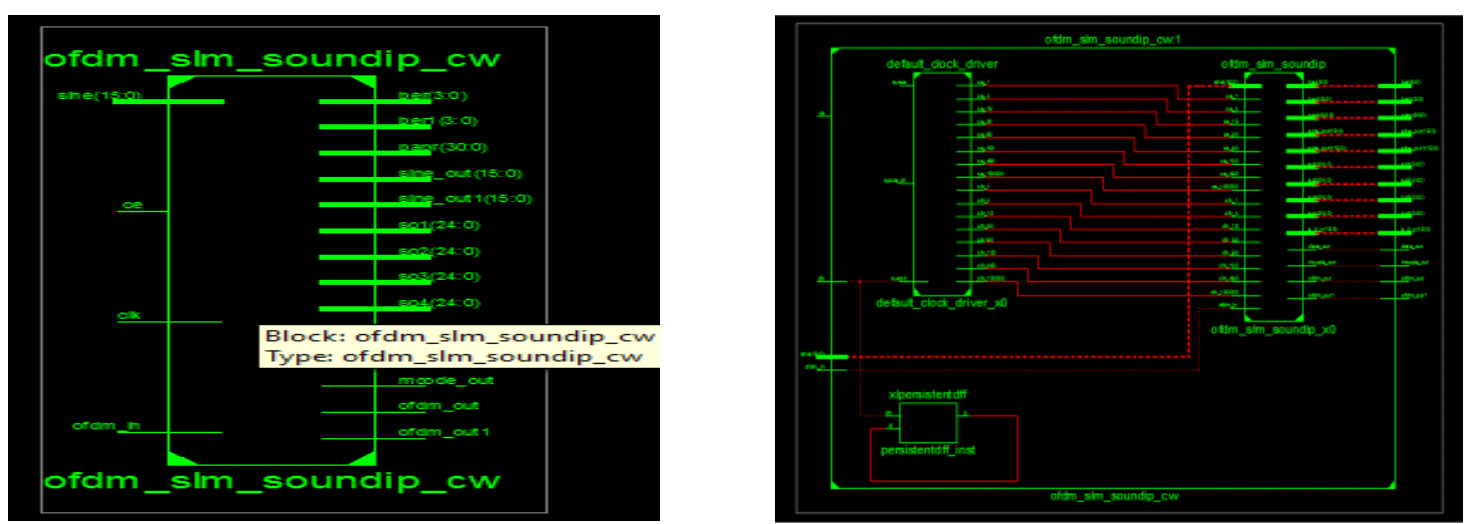

Fig 5.6 RTL schematic
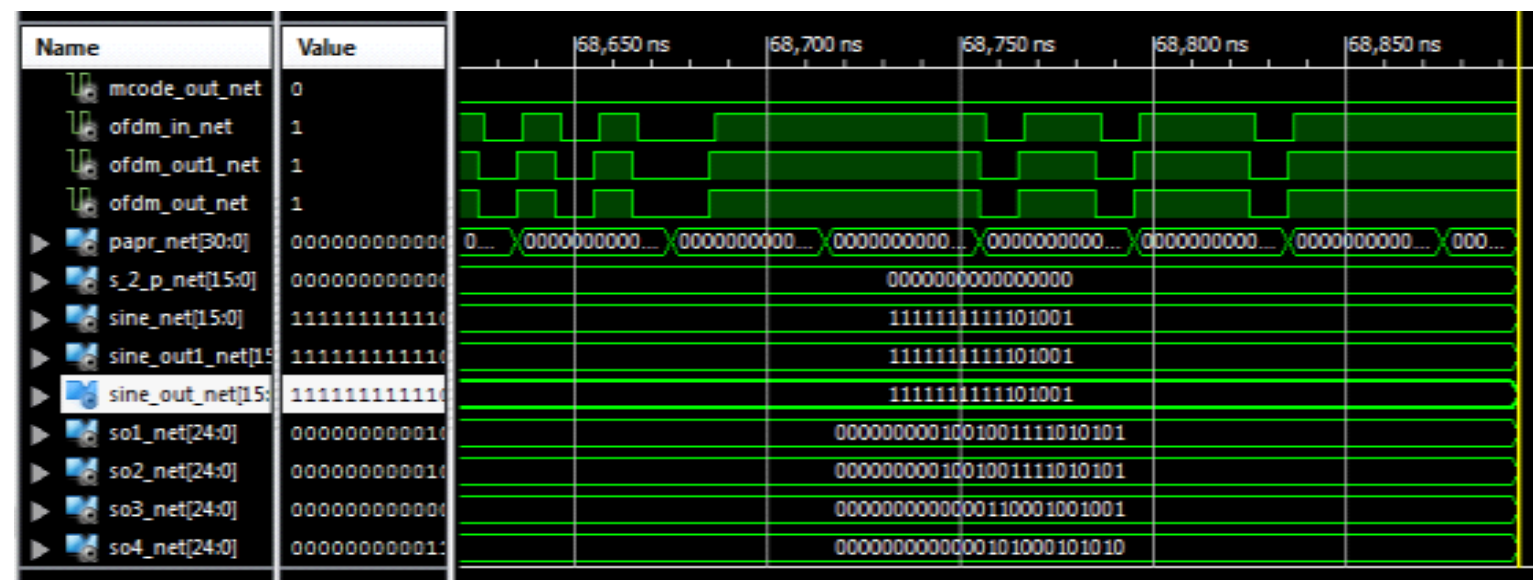

Fig 5.7 Test Bench 


\section{Conclusion}

OFDM is a very attractive technique for wireless communications. One of the serious drawbacks of OFDM is its high PAPR when the input sequences are highly correlated. The PAPR obtained without SLM for OFDM system is $12 \mathrm{~dB}$ and with SLM is $10 \mathrm{~dB}$ resulting in overall reduction of $2 \mathrm{~dB}$. Thus SLM technique has the potential to reduce PAPR for OFDM systems and improve its performance in terms of low PAPR high SNR and improved BER.

Xilinx System Generator combined with Matlab Simulink provides an easier and efficient way of developing the FPGA system design and simulating it. Also the hardware co-simulation feature of the software enables easier way to test and debug the design effectively on the actual hardware

The hardware co-simulation, RTL Schematics, Test Bench and VHDL codes, are also obtained for the implemented OFDM system. First the model is created in the Matlab/Simulink environment. The Matlab Simulations were carried out and necessary modifications were done. The hardware co-simulation was done to run the model on the hardware platform. The VHDL code, RTL Schematics and Test Bench were generated for the model. Test Vectors are passed through the test bench to verify functionality of the each block of the model. At the end complete functionality of the model is verified using Matlab Simulations, Test Bench/Modelsim Simulations and Hardware cosimulations.

\section{FUTURE SCOPE}

The paper deals with reduction of PAPR in a SISO OFDM. The concept of reduction of PAPR can be implemented in MIMO technology. If such a model is implemented it shall give the advantages of low PAPR as well as high data rate, which is an advantage of MIMO.

Different modulation techniques such as QPSK or QAM accommodating number of subcarriers as 1024 or up to 4096 can be experimentally tried to achieve data rates in multiple of $100 \mathrm{Mbps}$ or more.

The new system model can emphasis on performance of Signal to Noise Ratio, Bit Error Rate, CDF and CCDF with respect to channel capacity.

\section{ACKNOWLEDGEMENT}

Our thanks to the experts who have contributed towards development of this paper.

\section{References}

[1] Mathuran Athan "Simulation of OFDM system in Matlab - BER Vs Eb/N0 for OFDM in AWGN channel" Gaussian Waves, http://www.gaussianwaves.com 2011/07/simulation-of-ofdm-system-in-matlab-ber-vs-ebn0-for-ofdm-in-awgn-channel/

[2] Zoha Pajoudi and Sied Hamidreza Jamali "Hardware Implementation of a 802.11n MIMO OFDM Transceiver", International Symposium on Telecommunications, 2008, pp. 414-419

[3] Yu Wei Lin and Chen Yi Lin, "Design of FFT/IFFT Processor for MIMO OFDM Systems", IEEE Transaction on circuit and system, vol. 54 , no. 4 , pp. $807-815,2007$

[4] Ashok Jhunjhunwala, "Next Generation wireless for rural areas", Indian Journal of Radio and Space Physics, vol. 36, pp. 165-167, 2007

[5] K. C. Chang and Gerald E. Sobelman "FPGA Based Design of a Pulsed-OFDM system," IEEE APCCAS ,2006, pp. 1128-1131

[6] N.V Irukrulapati,V.K Chakka and A. Jain "SLM based PAPR reduction of OFDM signal using new phase sequence",electronic letters, vol45 No24, 2009.

[7] V.Vijayarangam,R.Kalidoss, Dr.(Mrs)R.Sukanesh “LCM for PAPR Reduction in OFDM systems”, ICWMMN Proceedings, 2006.

[8] N.V Irukulapati, V.KChakka and A.Jain "SLM based PAPR reduction of OFDM signal using new phase sequence" electronic letters, vol 45 No.24.

[9] Xiaowen Gu, Seungmin Baek, Suwon Park "PAPR Reduction of OFDM Signal Using an Efficient SLM Technique, 2007, LTE Solution Department, Shanghai Huawei Technologies Co., Ltd, Shanghai, China

[10] R. van Nee and A. deWild, "Reducing the peak to average power ratio of OFDM," in Proceedings of the 48th IEEE Semiannual Vehicular Technology Conference, May 1998, vol. 3, pp. 2072-2076.

[11] Dae-Woon Lim, Jong-Seon No "A New SLM OFDM Scheme With Low Complexity for PAPR Reduction" 2005, IEEE Signal Processing Letters, Vol. 12, No 2 .

[12] T. Jiang, M. Guizani, H. Chen, W. Xiang, and Y. Wu, "Derivation of PAPR distribution for OFDM wireless systems based on extreme value theory," IEEE Transactions on Wireless Communications, 2008.

[13] Hujun Yin and Sivash Alamouti, " OFDMA: A Broadband wireless Access Technology," Intel Corporation, 2008

[14] Dov Wulich and Gill. R. Tsouri "Is PAPR reduction always justified for OFDM , Ben-Gurion University of the Negev.

[15] Er.Sukhjinder Singh, Er. Jagjit Malhotra,Er. Manwinder Singh ,"A Novel SLM based PAPR Reduction Technique in OFDM-MIMO System : International Journal of Computers \& Technology, vol 2.

[16] G. L. Ren, H. Zhang, and Y. L. Chang, "A complementary clipping transform technique for the reduction of peak-to-average power ratio of OFDM system," IEEE Transactions on Consumer Electronics, vol. 49, no. 4, pp. 922-926, Nov. 2003.

[17] H. Ochiai and H. Imai, "On the distribution of the peak-to-average power ratio in OFDM signals" IEEE Transactions on Communications, vol. 49, no. 2, pp. 282-289, Feb. 2001.

[18] Mohammed I. Asseri, Charaslampos C. Tsimenidis, Bayan S. Sharif \& Stephane Y. Le Goff "Orthogonal - SLM technique for PAPR reduction \& Recovery without side information in OFDM systems, in IEEE transactions.

[19] Charan Langton, “Orthogonal Frequency Division Multiplexing (OFDM) Tutorial”,http://zoo.cs.yale.edu/classes/cs434/cs434-2012fall/readings/ofdm2.pdf

[20] Xilinx, "System Generator for DSP, User Guide", http://www.xilinx.com/documentation/sw manuals/xilinx14_1/sysgen user.pdf

[21] Hyun-Bae Jeon, Dong-Joon Shin, Dae Woon, "Partial Bit Inverted SLM Scheme for PAPR Reduction in QAM Modulated OFDM Signal" ,2009,IEEE transactions.

[22] TIan Ming Ma, Yu Song Shi \& Ying - Guan Wang, “A Novel SLM Scheme for PAPR Reduction in OFDM systems”, Journal of Computer Networks \& Communications .

[23] "Wireless communication"- Upena Dalal, $3^{\text {rd }}$ Impression,2010, Oxford publication

[24] K. C. Chang, et. al., "FPGA Based Design of a Pulsed-OFDM system," in IEEE Jour. 1-4244-0387-1/06. 